Doi: $10.15863 /$ TAS

International Scientific Journal

\section{Theoretical \& Applied Science}

p-ISSN: 2308-4944 (print) e-ISSN: 2409-0085 (online)

Year: $2014 \quad$ Issue: $11 \quad$ Volume: 19

Published: $30.11 .2014 \quad$ http://www.T-Science.org
Sergey Alexandrovich Mishchik Associate Professor,

Candidate of Pedagogical Science, Assistant professor Department of Physics, State Maritime University Admiral Ushakov, Russia, sergei mishik@mail.ru

SECTION 21. Pedagogy. Psychology. Innovation in Education.

\title{
MATHEMATICAL MODELING INTEGRITY - SYSTEM PERFORMANCE SUBJECT - FOURTH TASK PEDAGOGOMETRIKS
}

Abstract: Suggested a holistic modeling - system stakeholder systematic methods of mathematical analysis, linear algebra and matrix representations regarding the integrity - the system based on the formation of personality psychology - pedagogical activity theory, psychological - pedagogical system analysis and the theory of the formation of mental actions.

Key words: pedagogometriks, consistency, integrity, stakeholders, personality analysis, estimated processes.

Language: Russian

Citation: Mishchik SA (2014) MATHEMATICAL MODELING INTEGRITY - SYSTEM PERFORMANCE SUBJECT - FOURTH TASK PEDAGOGOMETRIKS. ISJ Theoretical \& Applied Science 11 (19): 51-54. doi: http://dx.doi.org/10.15863/TAS.2014.11.19.10

\section{УДК 372.851}

\section{МАТЕМАТИЧЕСКОЕ МОДЕЛИРОВАНИЕ ЦЕЛОСТНО-СИСТЕМНОГО СУБЬЕКТА ДЕЯТЕЛЬНОСТИ - ЧЕТВЁРТАЯ ЗАДАЧА ПЕДАГОГОМЕТРИКИ}

Аннотация: Предложено моделирование иелостно-системного субъекта деятельности методами математического системного анализа, линейной алгебры и матричных представлений относительно целостно-системного формирования личности на основе психолого-педагогической теории деятельности, психолого-педагогического системного анализа и теории формирования умственных действий.

Ключевые слова: педагогометрика, системность, иелостность, субъект деятельность, личность, анализ, ориентирочные проиессы.

Проблема математического моделирования целостно-системного субъекта деятельности направлена на дальнейшее аналитическое развитие психологической теории деятельности, психолого-педагогического системного анализа и теории формирования интеллекта средствами функционального анализа, дискретной математики, математической логики, теорией операций, вероятностными методами, теорией игр, теорией прогнозирования, а также формируемыми методами макро- и микропедагогометрики. Совместное применение выделенных психолого-дидактикоматематических средств определяет четвёртую задачу педагогометрики - математическое моделирование целостно-системного субъекта деятельности[1,2].

Целостно-системный

субъект жизнедеятельности (ЦССЖ) представляет единство предметных и деятельностных отношений и некоторую совокупность изменяющихся во времени функций, имеющих одни и те же свойства - детерминированные неслучайные процессы и стохастические случайные процессы. Можно определить группу процессов субъектных отношений, которые составляют абсолютно интегрируемые функции, для которых выполняется условие $\int_{0}^{\infty} X(t) d t<\infty$

Выделяется группа целостно 
реализуемых процессов. К ним относятся процессы с ограниченными внешними параметрами целостно-личностных характеристик. Для них $\int_{0}^{\infty} X^{2}(t) d t<\infty$. Эти процессы обозначим как квадратичные формы $L^{2}(t)$. Основные задачи математического проектирования ЦССЖ сводятся к установлению меры различения предметных и деятельностных процессов и определение способов их развития [3].

Поэтому устанавливаем пространство целостно-системных процессов как множество состояний субъекта, обладающего каким-либо базисным свойством, дополненное условием различения системной ориентировки. Пусть мерой различения процессов является интеллектуальное расстояние, которое фиксируется на числовой оси, отображающей типы ориентировочной деятельности. Расстояние между процессами определяется метрикой, обозначаемой $d(X, Y)=\mathrm{d}[X(t), Y(t)]$, где $X(t), Y(t)$ - ориентировочные процессы[4,5]

Это позволяет сформировать метрическое пространство как множество, в котором задано расстояние между каждыми двумя элементами ориентировочного состояния субъекта в виде действительной функции $d(X, Y)$, удовлетворяющей трем аксиомам: 1) $d(X, Y)=d(Y, X)$ $d(X, Y)+d(Y, Z) \geq d(X, Z)$.

Понятие метрики определяется внутренними (системными) свойствами ЦССЖ. Существуют функции от $X, Y$, которые могут быть взяты в качестве трёх метрик ориентировочно-исполнительно-контрольного уровня:

и

$$
d_{1}=\int_{t}|X(t)-Y(t)| d t \quad d_{2}=\sqrt{\int_{t}|X(t)-Y(t)|^{2} d t} ;
$$

$$
d_{3}=\sup |X(t)-Y(t)|
$$

ЦССЖ представляет пространство двенадцати базисных состояний, которые образуют метрическое пространство, каждый элемент которого полностью определяет поведение субъекта.

ЦССЖ в пространстве состояний, разделяется на взаимосвязанные подсистемы уровней: деятельности, действия и операции. Пространство субъектности представляется совокупностью субпространств состояний (сечений пространства личности). Элементы пространства состояний субъекта устанавливают конечномерные совокупности действительных чисел - векторы состояния личности $X=\left[\begin{array}{ll}x & , \ldots, x\end{array}\right]^{\mathrm{T}}$. Системы личности задаются векторами: $X(t)=[x(t), \ldots, x(t)]^{T} ; X(k)=[x$ $(k), \ldots, x(k)]^{T}$, где $T$ - знак транспонирования $[6,7]$.

Элементами евклидова личностного пространства состояний являются векторы $X=\left[x_{1}, \ldots, x_{n}\right]^{T}$, для которых уровень субъектного роста выражается формулой

$$
d\left(X^{\prime}, X^{\prime \prime}\right)=\sqrt{\sum_{j=1}^{n}\left(x_{j}^{\prime}-x^{\prime \prime}\right)^{2}}
$$

Возникающее линейное пространство ЦССЖ формирует возможность производить над ними действия аддитивного и мультипликативного преобразования $X+Y, a X$, где $X, \quad Y \quad$ - процессы; $a$-скалярный коэффициент. При этом выполняются основные свойства линейной алгебры:

1) $X+Y=Y+X$

2) $(X+Y)+Z=X+(Y+Z)$;

3) если $X+Y=X$, то $Y=0$ ( $Y$ - нулевой вектор);

4) $a(X+Y)=a X+a Y \quad(a-\quad$ скалярный коэффициент);

5) $a b X=b(a X) \quad(a, \quad b \quad-\quad$ скалярные коэффициенты);

6) $(a+b) X=a X+b X$.

Пространство преобразования ЦССЖ создают линейное пространство личности (ЛПЛ). Возникающие вектора личностных характеристик $X 1, \ldots, X_{n}$ устанавливают линейно независимую подсистему, через которую выражаются все $X_{1}, \ldots, X_{n}$. и создают базисные векторы, из которых формируется субъектная линейная комбинации векторов $\sum_{j=1}^{n} a_{j} X_{j}$,где $a_{j}$ скалярные коэффициенты. Условие линейной независимости субъектных векторов выражается через линейную комбинацию $\sum_{j=1}^{n} a_{j} X_{j}=0 \quad$ только при $a_{j}=0$. Условие линейной независимости системной характеристики личности $X_{j}$ определяется и через определитель Грамма (D): $D=\left|\begin{array}{l}\left(X_{1}, X_{1}\right) \ldots\left(X_{1}, X_{n}\right) \\ \left(X_{n}, X_{1}\right) \ldots\left(X_{n}, X_{n}\right)\end{array}\right| \neq 0$, где $\left(X_{i}, X_{j}\right)$ - скалярное произведение субъектных векторов.

Скалярным произведением развития личностных процессов $X_{i}(t), X_{j}(t)$ является выражение

$$
\left(X_{i}, X_{j}\right)=\int_{0}^{\infty} X_{i}(t) X_{j}^{*}(t) d t
$$

где $X_{j}^{*}$ - комплексно-сопряженный личностный вектор.

Свойства субъектного скалярного произведения:
1) $(a X+b Y, Z)=a(X, Z)+b(Y, Z)$;
2) $(X, X) \geq 0$; 
3) $(X, Y)=(Y, X)^{*}, \quad$ определяют следствия $(a X, Y)=a(X, Y), \quad(X, a Y)=a *(X, Y)$.

Скалярное субъектное произведение $(X, Y)$ при дискретных процессах развития личности $X(k)=[x(1), \ldots, x(n)]^{\mathrm{T}}, Y(k)=[y(1), \ldots, y(n)]^{T}$

вычисляется через сумму

$$
(X, Y)=\sum_{k=1}^{n} x(k) y(k)
$$

или в матричном виде $(X, Y)=X^{T}(k) Y(k)$.

Инвариантная норма личностного процесса $\mathrm{X}(\mathrm{t})$ определяется соотношением $\|\mathrm{X}\|=(\mathrm{X}, \mathrm{X})^{1 / 2}$.

Существуют свойства субъектной нормы:

1) $\|X\| \geq 0$;

2) $\|\mathrm{X}+\mathrm{Y}\| \leq\|\mathrm{X}\|+\|\mathrm{Y}\|$;

3) $\|\mathrm{aX}\|=|\mathrm{a}|\|\mathrm{X}\|$.

Первое свойство личностной нормы следует из условия $(\mathrm{X}, \mathrm{X})^{0,5} \geq 0$. Второго свойство отражает неравенство Шварца $|(X, Y)|^{2}$ $\leq(\mathrm{X}, \mathrm{X})(\mathrm{Y}, \mathrm{Y})$.

Связь метрического и линейного субъектно-личностного пространств следует из того, что норма разности двух ориентировочных процессов обладает свойствами метрики, т.е. является метрикой $\|\mathrm{X}-\mathrm{Y}\|=\mathrm{d}_{2}(\mathrm{X}, \mathrm{Y})$. Свойство метрики $\mathrm{d}_{2}(\mathrm{X}, \mathrm{Y})=\mathrm{d}_{2}(\mathrm{Y}, \mathrm{X})$ определяется, так как $\|\mathrm{X}-\mathrm{Y}\|=\|-(\mathrm{Y}-\mathrm{X})=\mid-1\| \mathrm{Y}-\mathrm{X}\|=\| \mathrm{Y}-\mathrm{X} \| . \quad$ Второе свойство ЦССЖ выполняется так как субъектная норма $\|X-Y\| \geq 0$ третьего свойства личностной метрики $\mathrm{d}_{2}(\mathrm{X}, \mathrm{Y})+\mathrm{d}_{2}(\mathrm{Y}, \mathrm{Z}) \geq \mathrm{d}_{2}(\mathrm{X}, \mathrm{Z})$. Тогда норма субъектной разности $\|\mathrm{X}-\mathrm{Y}\|+\| \mathrm{Y}-$ $\mathrm{Z}\|\geq\| \mathrm{X}-\mathrm{Z} \| . \quad$ Учтем $\quad\|\mathrm{X}-\mathrm{Z}\|=\|(\mathrm{X}-\mathrm{Y})+(\mathrm{Y}-\mathrm{Z})\| \quad$ и используем второе свойство личностной нормы $\|(\mathrm{X}-\mathrm{Y})+(\mathrm{Y}-\mathrm{Z})\| \leq\|\mathrm{X}-\mathrm{Y}\|+\|\mathrm{Y}-\mathrm{Z}\|$. Свойства личностной метрики для $\|\mathrm{X}-\mathrm{Y}\|$ выполняются, и поэтому линейное пространство личности является метрическим $[8,9]$.

При математическом моделированнии ЦССЖ выделим ортогональные личностные процессы, скалярное произведение которых равно нулю: $(\mathrm{f}, \mathrm{f})=0$. Для ортонормированных личностных процессах $\left(\mathrm{f}_{\mathrm{i}}, \mathrm{f}_{\mathrm{j}}\right)=1$. Матричная форма ортонормированного личностного базиса имеет вид $F^{\mathrm{T}} F=I$, где $\mathrm{F}=\left[\mathrm{f}{ }_{1}(\mathrm{t}), \ldots, \mathrm{f}_{\mathrm{n}}(\mathrm{t})\right] ; \quad \mathrm{I}-$ единичная матрица.

В математическом моделировании можно показать, что ортонормированная система субъектных функций является базисом личностных процессов. Личностный базис предполагает линейную независимость подсистемы субъектных функций $\mathrm{F}=\left(\mathrm{f}_{1}, \ldots, \mathrm{f}_{\mathrm{n}}\right)$, a условие линейной независимости векторов устанавливается через определитель Грамма $(\mathrm{D} \neq 0)$. Определитель $\mathrm{D}=\left|\mathrm{F}^{\mathrm{T}} \mathrm{F}\right|$ в данном случае равен 1 , так как для ортонормированной системы функций выполняется $F^{\mathrm{T}} F=I$.

Представление субъектных процессов через базисные личностные функции

$$
X(t)=\sum_{i=1}^{n} a_{i} f_{i}(t)
$$

где $a_{i}$ - скалярные коэффициенты, или в матричной форме $\mathrm{X}=\mathrm{FA}$, где $\quad \mathrm{F}=\left[\mathrm{f}_{1}(\mathrm{t}), \ldots, \mathrm{f}_{\mathrm{n}}(\mathrm{t})\right] ; \quad \mathrm{A}=\left(\mathrm{a}_{1}, \ldots, \mathrm{a}_{\mathrm{n}}\right)^{\mathrm{T}}$ позволяет определять коэффициенты $a_{i}$ в компактной матричной форме. В случае, если личностный базис $\mathrm{F}$ является ортонормированным, умножим последнее соотношение на $\mathrm{F}^{\mathrm{T}}$ слева: $\mathrm{F}^{\mathrm{T}} \mathrm{X}=\mathrm{F}^{\mathrm{T}} \mathrm{FA}$. Тогда $\mathrm{A}=\mathrm{F}^{\mathrm{T}} \mathrm{X}$. Произведение $\mathrm{F}^{\mathrm{T}} \mathrm{X}$ является вектором субъектных скалярных произведений

$$
A=\left(\begin{array}{l}
\left(X, f_{1}\right) \\
\left(X, f_{n}\right)
\end{array}\right)
$$

где коэффициенты $a_{j}$ рассчитывают по формуле $a_{j}=\int_{0}^{\infty} X(t) f_{j}^{*}(t) d t$.

Если $F^{\mathrm{T}} F \neq 1, \quad$ то для вычисления вектора целостно-системного субъекта А имеем формулу $A=\left(F^{\mathrm{T}} F\right)^{-1} F^{\mathrm{T}} X$. Обратная личностная матрица $\left(F^{\mathrm{T}} F\right)^{-1}$ определяется по функции Грамма:

$$
\left(F^{\mathrm{T}} F\right)=\left[\begin{array}{l}
\left(f_{1}, f_{1}\right) \ldots\left(f_{1}, f_{n}\right) \\
\ldots \\
\left(f_{n}, f_{1}\right) \ldots\left(f_{n}, f_{n}\right)
\end{array}\right] .
$$

Представленное математическое моделирование целостно-системного субъекта жизнедеятельности устанавливает четвёртую задачу педагогометрики, направленную на функциональное соотношение предметных и деятельностных основ специалиста широкого профиля.

\section{References:}

1. Mishchik SA (2011) Proektirovanie matematicheskikh modeley fizicheskikh ob'ektov v protsesse formirovaniya tselostnosistemnoy samostoyatel'noy uchebnoy deyatel'nosti. Odinnadtsataya mezhdunarodnaya konferentsiya - Fizika v sisteme sovremennogo obrazovaniya (FSSO 11), 1 tom - Volgograd: Izd-vo VGPU, 318.
2. Mishchik SA (2012) Organizatsiya laboratornogo fizicheskogo praktikuma na baze mobil'nykh programm platformy android $\mathrm{v}$ protsesse tselostno-sistemnoy shirokoprofil'noy podgotovki. XII Mezhdunarodnaya uchebno-metodicheskaya konferentsiya - Sovremennyy fizicheskiy 
praktikum, Moscow, 25-27 sept. 2012, Izd-vo MGTU im. N.E. Baumana, 325.

3. Mishchik SA (2014) Tselostno-sistemnyy tsikl uchebnoy zhiznedeyatel'nosti - model' professional'noy deyatel'nosti shirokoprofil'nogo spetsialista. Materialy Mezhdunarodnoy nauchnoy konferentsii «Deyatel'nostnaya teoriya ucheniya: sovremennoe sostoyanie i perspektivy», Moscow 6-8 febr. 2014, Izdatel'stvo Moskovskogo universiteta, 384.

4. Mishchik SA (2014) Bazisnost'. Fundamental'nost'. Shirokoprofil'nost'. Pedagogometrichnost'. Materialy Mezhdunarodnoy nauchnoy konferentsii «Moderni vymozenosti vedy - 2014». - Dil 16. Pedagogika.: Praha. Publishing House «Education and Science» S.r.o, 112.

5. Mishchik SA (2014) Modelirovanie shirokoprofil'noy tselostno-sistemnoy deyatel'nosti. Materialy II Mezhdunarodnoy nauchnoy konferentsii «Prioritety mirovoy nauki: eksperiment i nauchnaya diskussiya»: 24-25 dec. 2013, S-Peterburg North Charleston, SC, USA: CreateSpace, 151.

6. Mishchik SA (2013) Formirovanie tselostnosistemnogo tsikla uchebnoy zhiznedeyatel'nosti shirokoprofil'nogo spetsialista metodami matematicheskogo modelirovaniya. Sbornik materialov 3-y mezhdunarodnoy nauchno-prakticheskoy konferentsii. 2 chast'. Problemy sovremennoy nauki v 21 veke (Makhachkala, 28 dec. 2013): - Makhachkala: OOO «Aprobatsiya», 195.

7. Mishchik SA (2014) Strukturnoe formirovanie pedagogometricheskikh funktsiy matematicheskogo analiza tselostnosistemnogo uchebnogo protsessa. Materialy Mezhdunarodnoy nauchnoy konferentsii «Nastoyashchi izsledvaniya i razvitie - 2014» 17-25 jan. 2014. Tom 14. Pedagogicheski nauki. Sofiya, «Byal GRAD-BG» OOD, 96.

8. Mishchik SA (2014) Pedagogometrika i matematicheskoe modelirovanie uchebnoy deyatel'nosti. Materialy Mezhdunarodnoy nauchnoy konferentsii «Modern mathematics in science» - 30.06.2014 Caracas, Venezuela. ISJ Theoretical \& Applied Science 06 (14): 54-56.

doi: http://dx.doi.org/10.15863/TAS.2014.06.14.10

9. Tokmazov GV (2014) Matematicheskoe modelirovanie $\mathrm{v}$ uchebno-professional'noy deyatel'nosti. Materialy Mezhdunarodnoy nauchnoy konferentsii «Modern mathematics in science» - 30.06.2014 Caracas, Venezuela. ISJ Theoretical \& Applied Science 06 (14): 44-46.

doi: http://dx.doi.org/10.15863/TAS.2014.06.14.8 